\title{
Epidemiological profile and obstetric outcomes of patients with peripartum congestive heart failure in Taiwan: a retrospective nationwide study
}

Ying-Jen Chang ${ }^{1}$, Chung-Han Ho ${ }^{2,3}$, Jen-Yin Chen ${ }^{1,4}$, Ming-Ping Wu ${ }^{5,6}$, Chia-Hung Yu' ${ }^{1}$, Jhi-Joung Wang ${ }^{1,2}$, Chia-Ming Chen ${ }^{1 *+}$ and Chin-Chen Chu ${ }^{1,2,7,8^{*}}$ (iD

\begin{abstract}
Background: During pregnancy, the hyperdynamic physiology of circulation can exacerbate many cardiovascular disorders. Congestive heart failure (CHF) usually occurs during late pregnancy, which is significantly associated with a high level of maternal and neonatal morbidities and mortalities. The profile of women who develop peripartum CHF (PCHF) is unknown. We investigated the epidemiological profiles of PCHF.

Methods: In this retrospective cohort study, PCHF patients were identified using International Classification of Diseases, Ninth Revision, Clinical Modification (ICD-9-CM) codes in Taiwan's National Health Insurance Research Database. Risk factors and obstetric outcomes were compared in women with and without PCHF.

Results: From 2,115,873 birth-mothers in Taiwan between 1997 and 2013, we identified 512 with PCHF (incidence: 24. $\left.20 / 10^{5}\right)$. More women with than without PCHF were older ( $\geq 35,18.16 \%$ vs. 9.62\%), and had more multifetal gestations (7.42\% vs. $1.40 \%)$, gestational hypertension (HTN) (19.2\% vs. 1.31\%), and gestational diabetes mellitus (4.10\% vs. $0.67 \%)$. After the analysis had been adjusted for confounders, the leading comorbidities associated with PCHF were structural heart diseases (adjusted odds ratio [aOR]: 67.21; 95\% confidence interval [Cl]: 54.29-83.22), pulmonary diseases (aOR: 13.12; 95\% Cl: 10.28-16.75), chronic HTN (aOR: 11.27; 95\% Cl: 6.94-18.28), thyroid disease (aOR: 9.53; 95\% Cl: 5.27-17.23), and gestational HTN (aOR: 5.16; 95\% Cl: 3.89-6.85). PCHF patients also had a higher rate of cesarean sections $(66.41 \%$ vs. $34.46 \% ; p<0.0001)$.

Conclusion: Maternal structural heart diseases, pulmonary diseases, thyroid disorders, and preexisting or gestational HTN are associated with a higher risk of developing PCHF. Birth-mothers with PCHF also had a higher risk of caesarean section and adverse outcomes, including maternal death. Our findings should benefit healthcare providers, and government and health insurance policy makers.
\end{abstract}

Keywords: Congestive heart failure, Peripartum, Epidemiological, Obstetric outcomes

\footnotetext{
* Correspondence: fcweden@gmail.com; chinchen.chu@gmail.com

${ }^{\dagger}$ Equal contributors

${ }^{1}$ Department of Anesthesiology, Chi Mei Medical Center, 901 Zhonghua

Road, Yongkang District, Tainan City 710, Taiwan

Full list of author information is available at the end of the article
}

(c) The Author(s). 2017 Open Access This article is distributed under the terms of the Creative Commons Attribution 4.0 International License (http://creativecommons.org/licenses/by/4.0/), which permits unrestricted use, distribution, and reproduction in any medium, provided you give appropriate credit to the original author(s) and the source, provide a link to the Creative Commons license, and indicate if changes were made. The Creative Commons Public Domain Dedication waiver (http://creativecommons.org/publicdomain/zero/1.0/) applies to the data made available in this article, unless otherwise stated. 


\section{Background}

Congestive heart failure (CHF) is a complicated clinical syndrome that impairs ventricular pumping [1]. During pregnancy, the hyperdynamic physiology of circulation can exacerbate many cardiovascular disorders. Peripartum CHF (PCHF) usually occurs during late pregnancy or within a few months postpartum and is often significantly associated with high levels of maternal and neonatal morbidity and mortality [2, 3]. PCHF is the most common major cardiovascular complication in pregnant women with a preexisting cardiac disease [4].

PCHF can be caused by exacerbating preexisting cardiovascular [4] and systemic diseases [5-7], or by pregnancy-associated diseases [8]. Sometimes, PCHF can be idiopathic; and in this situation, it is referred to as peripartum cardiomyopathy (PPCM) [9, 10]. No matter what the etiology, PCHF is associated with adverse outcomes for birth-mothers and neonates [11]. The incidence of peripartum cardiovascular disease is growing [12], most likely because of older maternal age [13], cardiovascular risk factors (e.g., obesity, diabetes mellitus $[\mathrm{DM}]$, and hypertension [HTN]), and the lifespan of women with congenital heart disease [14].

There are many studies on PPCM, but studies on PCHF are still scarce. In addition, the epidemiological reports that have been published are either small-scale studies or based on small community databases $[15,16]$. For such a critical disease, the existing literature is insufficient. Therefore, to help fill this gap, we used a largescale national population-based database to investigate the long-term epidemiological profile of PCHF and determine its incidence, characteristics, and outcomes in the Taiwan population.

\section{Methods}

\section{Data source}

Taiwan launched a single-payer National Health Insurance (NHI) program on March 1, 1995 [17, 18]. The enrollment rate of NHI reached $99 \%$ in 1997, and has maintained at this high coverage level ever since [17]. The NHI research database (NHIRD) provides encrypted patient identification numbers, gender, date of birth, dates of admission and discharge, the ICD-9-CM (International Classification of Diseases, Ninth Revision, Clinical Modification) codes of diagnoses and surgical procedures, details of prescriptions, and costs covered and paid for by the NHI.

In Taiwan, every hospital has a coder-team to ensure the accuracy of diagnostic and management codes. Besides, Taiwan's NHI Bureau is responsible for auditing medical payments by comprehensive review of medical records, examination reports, and results of imaging studies. If physicians fail to meet the standards for clinical practice, Taiwan's NHI reserves the right to reject payment and can impose huge financial penalties.
We used the inpatient claims database from 1997 to 2013 because, in Taiwan, almost all deliveries occur in hospitals and almost all patients with severe diseases like heart failure are hospitalized. The dataset was released with de-identified secondary data for public research. The Taiwan National Health Research Institutes approved the present study. Moreover, because all types of personal identification were encrypted to secure patient privacy, the present study was granted an exemption from a full ethical review by the Chi Mei Medical Center Institutional Review Board (IRB: 10,505-014).

\section{Selection of patients and variables}

To ensure that patients with PCHF would be comparable with patients with PPCM, we defined PCHF patients as those with newly developed and diagnosed CHF (ICD-9-CM code 428.0-428.21) between 1 month before and 5 months after delivery, based on the definition of peripartum patients in an established article on PPCM [19]. Because we focus on the new onset of PCHF, we excluded pregnant women with a history of $\mathrm{CHF}$ or an onset of CHF out of this defined time frame (Fig. 1).

We recorded the maternal demographic factors: age, economic status, hospital type (medical center: 10002500 beds/tertiary care; regional hospital: 301-999 beds/ secondary care; or local hospital: < 300 beds/primary care); delivery-related factors: multifetal gestations, gestational HTN, gestational DM, and placental abnormality; comorbidities: HTN, congenital heart disease, DM, pulmonary disease, hepatic disease, renal disease, thyroid disease, anemia, malignancy, and autoimmune disease. Critical adverse cardiac events, such as ventricular tachycardia, ventricular fibrillation, and cardiac arrest, were also studied. Major comorbidities, obstetric conditions, and corresponding ICD-9-CM Codes are listed in Additional file 1.

Patients were also divided into three economic status groups based on monthly income in New Taiwan dollars (NT\$): < NT\$20,000; NT\$20,000-NT\$40,000; and > NT\$40,000.

\section{Epidemiological profile and maternal outcomes of PCHF} We determined the incidence of PCHF between 1997 and 2013. Next, we characterized the demographics and medical conditions of the patients and estimated the associations between PCHF and possibly important related obstetrical complications: maternal death, cardiac arrest, life-threatening arrhythmia, and acute pulmonary edema.

\section{Statistical analysis}

Significant differences in prevalence between different age groups were evaluated using a $X^{2}$ test. Odds ratios 


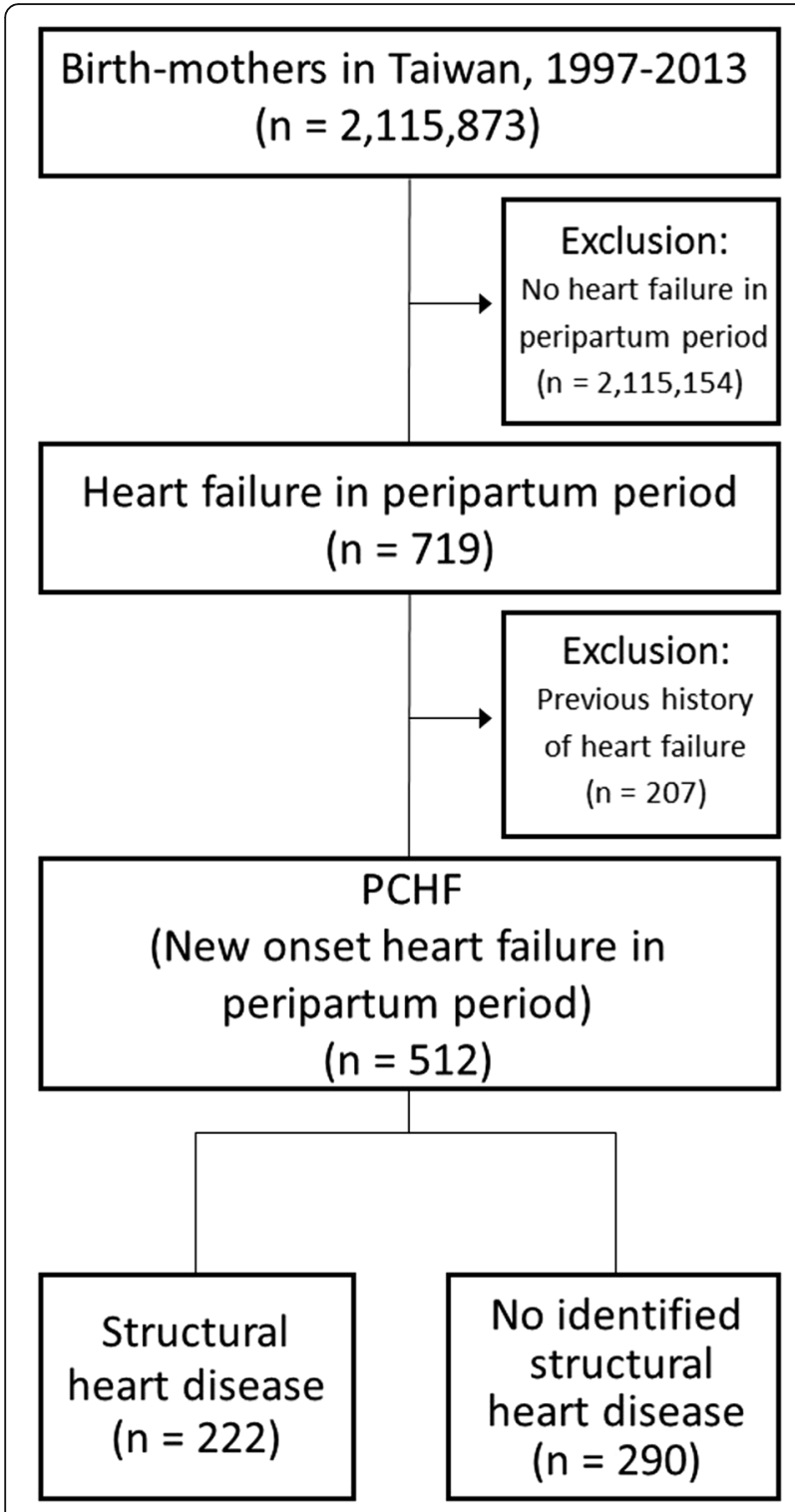

Fig. 1 Flow chart of the creation of the study sample. Description of data: We defined peripartum congestive heart failure (PCHF) as newly developed and diagnosed CHF (ICD-9-CM code: 428.0-428.21) between 1 month before and 5 months after delivery. Women with a history of $\mathrm{CHF}$ or an onset of CHF beyond the peripartum period were excluded

and $95 \%$ confidence intervals (ORs $\pm 95 \% \mathrm{CIs}$ ) for PCHF with and without each maternal comorbidity were determined using multivariate logistic regression adjusted for possible confounding factors: structural heart disease, pulmonary disease, liver disease, renal disease, thyroid disease, anemia, malignancy, autoimmune disease, chronic HTN, chronic DM, multifetal gestations, placental abnormality, gestational HTN, gestational DM, peripartum hemorrhage, hospital type, and economic status by monthly income. Each comorbidity was considered a single variable and was separately entered into the logistic regression model.
SAS 9.4 (SAS Institute Inc., Cary, NC, USA) was used for all data analyses. Significance was set at $p<0.001$ (two-sided).

\section{Results}

We identified 2,115,873 birth-mothers during 19972013 in Taiwan: $512(0.0242 \%)$ patients had been diagnosed with PCHF 1 month before and within 5 months after delivery. The incidence of PCHF was 1:4133 deliveries. Birth-mothers with PCHF were significantly $(p<0.001)$ older ( $\geq 35$ years old) than were birthmothers without PCHF (18.16 vs. 9.62\%). Additionally, they had significantly $(p<0.001)$ more comorbidities: structural heart disease $(43.36 \%$ vs. $0.40 \%)$, pulmonary disease (27.93 vs. $0.34 \%$ ), liver disease (2.73 vs. $0.09 \%$ ), renal disease ( 3.71 vs. $0.03 \%)$, thyroid disease (3.32 vs. $0.10 \%)$, anemia (16.21 vs. $2.62 \%)$, malignancy (2.34 vs. $0.61 \%)$, autoimmune disease (2.93 vs. $0.06 \%)$, chronic HTN (6.64 vs. $0.05 \%)$, and DM (2.15 vs. $0.11 \%)$ (Table 1).

Significantly $(p<0.001)$ more birth-mothers with PCHF had multifetal gestations (7.42 vs. $1.40 \%)$, gestational DM (4.10 vs. $0.67 \%)$, and gestational HTN (19.92 vs. $1.31 \%$ ), than did birth-mothers without PCHF (Table 1). However, there were no significant differences in placental abnormalities or peripartum hemorrhage between these two groups. Birth-mothers with PCHF earned significantly $(p<0.001)$ lower monthly incomes than did birth-mothers without PCHF. Moreover, significantly $(p<0.001)$ more women with PCHF sought care in medical centers $(25.20$ vs. $12.84 \%)$ than did women without PCHF (Table 1).

Comorbid structural heart disease raised the risk of PCHF to 190.58 times normal, and even after the analysis had been adjusted for age, hospital levels, monthly income, maternal comorbidities, and obstetric conditions, the risk was 67.21 times normal (Table 2). Comorbid pulmonary disease raised the risk of PCHF to 13.12 times normal, chronic HTN raised it to 11.27 times normal, and gestational HTN to 5.16 times normal. However, gestational DM, placental anomalies, and peripartum hemorrhage did not increase the risk (Table 2).

Only $290(0.01 \%)$ patients without a structural heart disease (reference group) vs. $222(2.56 \%)$ with a structural heart disease developed PCHF (Table 3). Among the structural heart diseases, right-side cardiac lesions raised the risk of PCHF to 70.65 times, compared with mothers who had no structural heart disease. Left-side cardiac lesions raised it to 60.00 times, and septal wall defects raised it to 33.95 times, compared with mothers had no structural heart disease (Table 3 ).

The risk of birth-mothers with PCHF developing major maternal adverse outcomes was high. For example, the risk ratio of acute pulmonary edema was 
Table 1 Characteristics of parturient women with and without peripartum congestive heart failure

\begin{tabular}{|c|c|c|c|}
\hline \multirow[t]{2}{*}{ Characteristics } & $\mathrm{PCHF}^{a}$ & No PCHF & \multirow[t]{2}{*}{$P$} \\
\hline & $n=512(\%)$ & $n=2,115,361(\%)$ & \\
\hline Age (years) & & & $<0.001$ \\
\hline$<20$ & $16(2.79)$ & $89,096(4.21)$ & \\
\hline $20-34$ & $403(77.91)$ & $1,822,741(86.17)$ & \\
\hline$\geq 35$ & $93(18.16)$ & $203,524(9.62)$ & \\
\hline \multicolumn{4}{|l|}{ Maternal comorbidities } \\
\hline Structural heart disease & $222(43.36)$ & $8463(0.40)$ & $<0.001$ \\
\hline Pulmonary disease & $143(27.93)$ & $7152(0.34)$ & $<0.001$ \\
\hline Liver disease & $14(2.73)$ & $1801(0.09)$ & $<0.001$ \\
\hline Renal disease & 19 (3.71) & $680(0.03)$ & $<0.001$ \\
\hline Thyroid disease & $17(3.32)$ & $2064(0.10)$ & $<0.001$ \\
\hline Anemia & $83(16.21)$ & $55,431(2.62)$ & $<0.001$ \\
\hline Malignancy & $12(2.34)$ & $12,984(0.61)$ & $<0.001$ \\
\hline Autoimmune disease & $15(2.93)$ & $1339(0.06)$ & $<0.001$ \\
\hline Chronic hypertension & $34(6.64)$ & $1026(0.05)$ & $<0.001$ \\
\hline Diabetes mellitus & $11(2.15)$ & $2316(0.11)$ & $<0.001$ \\
\hline \multicolumn{4}{|l|}{ Obstetric condition } \\
\hline Multifetal pregnancies & $38(7.42)$ & $29,697(1.40)$ & $<0.001$ \\
\hline Placenta abnormality & $10(1.95)$ & $23,252(1.10)$ & 0.064 \\
\hline Gestational HTN & $102(19.92)$ & $27,737(1.31)$ & $<0.001$ \\
\hline Gestational diabetes mellitus & $21(4.10)$ & $14,101(0.67)$ & $<0.001$ \\
\hline Peripartum hemorrhage & $24(4.69)$ & $51,363(2.43)$ & $<0.001$ \\
\hline Hospital type & & & $<0.001$ \\
\hline Medical center & $129(25.20)$ & $271,513(12.84)$ & \\
\hline Regional hospital & $106(20.70)$ & $412,589(19.50)$ & \\
\hline Local hospital & $277(54.10)$ & $1,431,199(67.66)$ & \\
\hline Monthly income (NT\$) ${ }^{b}$ & & & $<0.001$ \\
\hline$\leq 20,000$ & $255(49.80)$ & $821,380(38.83)$ & \\
\hline $20,000-40,000$ & $194(37.89)$ & $915,011(43.26)$ & \\
\hline$>40,000$ & $63(12.30)$ & $378,970(17.92)$ & \\
\hline
\end{tabular}

Characteristics of birth-mothers in Taiwan with and without peripartum congestive heart failure during 1997-2013

a PCHF: peripartum congestive heart failure

${ }^{\mathrm{b}} \mathrm{NTS}$ : New Taiwan dollar

826.31 times for mothers with than without PCHF (Table 4). Finally, cesarean sections were more frequent, hospital stays were longer, and hospitalization costs were all significantly higher for birth-mothers with PCHF (Table 5).

\section{Discussion}

We found that delivering mothers who are $\geq 35$ years old and who have preexisting comorbidities, especially structural heart disease and pulmonary disease, have a significantly higher incidence of PCHF. Moreover, women with multifetal gestations and gestational HTN were associated with a higher risk of developing PCHF than were normotensive mothers and the delivering mothers of singleton children. Additionally, delivering mothers with PCHF had a higher incidence rate of cesarean section, and they were more frequently associated with adverse outcomes-e.g., pulmonary edema, lifethreatening arrhythmia, episodes of cardiac arrest, and death-than were delivering mothers without PCHF.

This is the first nationwide epidemiological study of PCHF patients. Because our data source was a 16 -year population-based database, the statistical power of our analysis is stronger than that of other reports with smaller study populations.

Of the 512 patients who had been diagnosed with PCHF, 290 had not being coded any structural heart disease. Most of them were highly suspected of having PPCM. Although they were not directly coded as having PPCM (ICD-9-CM code 674.5), by definition they met the criteria for the diagnosis of PPCM [20]. We checked 2 recent PPCM cases in our hospital registration record and found they had been coded as CHF (ICD-9-CM codes 428.0-428.21), not PPCM (ICD-9-CM code, 674.5). They had been transferred to Cardiology after they had been diagnosed with heart failure. Therefore, they were coded CHF on discharge. The possible explanation is that cardiologists are more familiar with the CHF code than with the PPCM code. However, this needs to be verified.

The incidence of PPCM is estimated to be 1 in 2500 to $4000(0.03 \%)$ total births in Canada, Europe, and the United States, $0.1 \%$ in South Africa, and $0.09 \%$ in Asia, and higher in less-developed countries like Nigeria (1\%) and Haiti $(0.33 \%)$ [21, 22]. However, the incidence of PCHF has never been reported. Using our large database, we first showed that the incidence of PCHF in Taiwan was $0.024 \%$ of total births, which is close to the incidence of PPCM in the United States. We could not calculate the precise incidence of PPCM in Taiwan from our present study. We could only indirectly estimate that the incidence is near or lower than $0.014 \%$, i.e., 290 highly suspected cases of PPCM in 2,115,873 pregnant women. This incidence is much lower than that of PPCM in the United States (0.1\%), but higher than it is in Japan (about 0.005\%) [23].

Advanced age is always a risk for many poor gestational outcomes [24]. In our study, the percentage of PCHF patients $\geq 35$ years old was twice that of women without PCHF. Being a delivering mother $\geq 35$ might lead to higher incidence rates of pre-eclampsia, eclampsia, and gestational DM, which have been identified as risk factors for heart failure during pregnancy. Moreover, older women have had a substantial increase in multifetal pregnancies because of contemporary reproductive techniques [25]. 
Table 2 Associations between peripartum congestive heart failure and comorbidities

\begin{tabular}{|c|c|c|}
\hline Characteristics & Crude OR ${ }^{\mathrm{a}}[95 \% \mathrm{Cl}]$ & Adjusted $\mathrm{OR}^{\mathrm{b}}[95 \% \mathrm{Cl}]^{\mathrm{c}}$ \\
\hline \multicolumn{3}{|l|}{ 1. Maternal comorbidities } \\
\hline Structural heart disease & $190.58(159.81-227.27)$ & $67.21(54.28-83.22)$ \\
\hline Pulmonary disease & $114.24(94.05-138.76)$ & $13.12(10.28-16.75)$ \\
\hline Liver disease & 32.99 (19.36-56.23) & $3.61(1.85-7.05)$ \\
\hline Renal disease & 119.85 (75.33-190.68) & $4.53(2.28-8.99)$ \\
\hline Thyroid disease & $35.16(21.64-57.13)$ & $9.53(5.27-17.23)$ \\
\hline Anemia & 7.19 (5.68-9.10) & $2.99(2.28-3.91)$ \\
\hline Cancer & $3.89(2.19-6.89)$ & $1.80(0.97-3.35)$ \\
\hline Autoimmune disease & 47.65 (28.43-79.86) & $2.33(1.08-5.01)$ \\
\hline Chronic hypertension & $146.58(102.96-208.68)$ & $11.27(6.94-18.28)$ \\
\hline Diabetes mellitus & $20.03(11.01-36.46)$ & $1.33(0.54-3.26)$ \\
\hline \multicolumn{3}{|l|}{ 2. Obstetric conditions } \\
\hline Multifetal pregnancies & $5.63(4.05-7.84)$ & $2.13(1.47-3.08)$ \\
\hline Placenta anomaly & $1.79(0.96-3.35)$ & $1.21(0.51-2.88)$ \\
\hline Gestational HTN & $17.40(13.92-21.75)$ & $5.16(3.89-6.85)$ \\
\hline Gestational diabetes mellitus & $6.37(4.12-9.87)$ & $1.02(0.55-1.90)$ \\
\hline Peripartum hemorrhage & $1.98(1.31-2.98)$ & $0.98(0.54-1.76)$ \\
\hline
\end{tabular}

Associations between peripartum congestive heart failure and maternal comorbidities and obstetric conditions

${ }^{a} O R$ : odds ratio

${ }^{\mathrm{b}}$ Adjusted for all confounding factors in Table 1

${ }^{\mathrm{C}} \mathrm{Cl}$ : confidence interval

Not surprisingly, delivering mothers in our study with underlying cardiac disease had a greater risk of developing PCHF, which is similar to the findings of other reports [4, 26]. After age and comorbidities had been adjusted for, women with pre-existing structural heart disease were still associated with the highest chances of developing PCHF. In our study, $2.56 \%$ of the patients with structural heart disease developed PCHF, but in the European Society of Registry of Pregnancy and Cardiac disease (ROPAC) study [4], 13.1\% with a structural heart disease did. The higher incidence of heart failure in the ROPAC study might be attributable to that study's having included heart-failure cases during the entire pregnancy rather than limiting it to peripartum period, as

Table 3 Incidence of peripartum congestive heart failure comorbid with Structural heart disease

\begin{tabular}{|c|c|c|c|c|c|}
\hline \multirow[t]{2}{*}{ Characteristics } & \multirow{2}{*}{$\begin{array}{l}\text { PCHF }^{\mathrm{a}} \\
n=512\end{array}$} & No PCHF & \multirow{2}{*}{$\begin{array}{l}\text { Total } \\
\text { birth-mothers }\end{array}$} & \multirow{2}{*}{$\begin{array}{l}\text { Cardiac } \\
\text { lesion-specific } \\
\text { PCHF frequency }\end{array}$} & \multirow{2}{*}{$\begin{array}{l}\text { Adjusted } \\
\operatorname{OR}^{c}(95 \% \text { Cl) }\end{array}$} \\
\hline & & $n=2,115,361$ & & & \\
\hline \multicolumn{6}{|c|}{ Structural heart disease } \\
\hline Yes & 222 & 8463 & 8685 & $2.56 \%$ & $67.21(54.28-83.22)$ \\
\hline No & 290 & $2,106,898$ & $2,107,188$ & $0.01 \%$ & Reference group \\
\hline \multicolumn{6}{|c|}{ Location of anomaly } \\
\hline Left side & 23 & 424 & 447 & $5.15 \%$ & $60.00(35.54-101.29)$ \\
\hline Right side & 27 & 436 & 463 & $5.83 \%$ & $70.65(43.27-115.35)$ \\
\hline Septal defect & 112 & 5897 & 6009 & $1.86 \%$ & $33.95(26.56-43.38)$ \\
\hline Others & 77 & 2064 & 2141 & $3.60 \%$ & $22.66(16.46-31.18)$ \\
\hline \multicolumn{6}{|l|}{ Types } \\
\hline Valvular & 76 & 1264 & 1340 & $5.67 \%$ & 83.79 (61.49-114.18) \\
\hline Non-valvular & 290 & 7199 & 7345 & $1.99 \%$ & $32.90(25.88-41.82)$ \\
\hline
\end{tabular}

Incidence of peripartum congestive heart failure for birth-mothers in Taiwan comorbid with specific cardiac anomalies during 1997-2013

${ }^{a} P C H F$ : peripartum congestive heart failure

${ }^{\mathrm{b}}$ Adjusted for all confounding factors in Table 1

'OR: odds ratio

${ }^{\mathrm{d}} \mathrm{Cl}$ : confidence interval 
Table 4 Major adverse outcomes of parturient women with and without peripartum congestive heart failure

\begin{tabular}{llll}
\hline Major maternal adverse outcomes & $\mathrm{PCHF}^{\mathrm{a}}$ & No PCHF & Relative risk (95\% Cl) $^{\mathrm{b}}$ \\
& $n=512(\%)$ & $n=2,115,361(\%)$ & No PCHF birth-mother as reference \\
\hline Acute pulmonary edema & $48(9.375)$ & $240(0.011)$ & $826.31(613.65-1112.67)$ \\
Cardiogenic shock & $7(1.367)$ & $35(0.002)$ & $826.31(368.75-1851.66)$ \\
Life threatening arrhythmia or cardiac arrest & $4(0.781)$ & $88(0.004)$ & $187.80(69.21-509.60)$ \\
Maternal death & $3(0.586)$ & $376(0.018)$ & $32.96(10.62-102.33)$ \\
\hline
\end{tabular}

Major maternal adverse outcomes of parturient women with and without peripartum congestive heart failure during 1997-2013 in Taiwan

${ }^{a} P C H F$ : peripartum congestive heart failure

${ }^{\mathrm{b}} \mathrm{Cl}$ : confidence interval

our study did. Our finding is closer to that of a metaanalysis [27] which reported that the frequency of heart failure was $4.8 \%$ in mothers with congenital heart disease. Except for this, the lower rate in our study might be related to differences in lifestyle, other medical conditions, or genetics [10]. Epidemiological studies [28, 29] reported that pregnant Asian women had a lower BMI, less gestational weight gain, and fewer incidences of preeclampsia and abnormal liver enzymes than did pregnant Caucasian women, all of which might contribute to the low incidence of both PCHF and PPCM in Asians.

We also found that $5.83 \%$ of delivering mothers with right-side cardiac lesions developed PCHF, but that $5.15 \%$ with left-side lesions and that $1.86 \%$ with a septal defect developed PCHF. This is somewhat different from the ROPAC study [4] finding that more delivering mothers with left-side lesions developed PCHF than did those with right-side lesions or a septal wall defect. In our study, after confounding factors had been adjusted for, delivering mothers with right-side lesions had a greater risk of developing PCHF (aOR: 70.65) than did those with left-side lesions or a septal wall defect, which is consistent with the findings of a prospective multicenter study in Canada [30] and a retrospective study in China [31].

Delivering mothers with valvular heart disease had a greater risk of developing PCHF than did those with cardiac lesions not involving heart valves. According to many studies [4], between 18.7 and $48.3 \%$ of pregnant women diagnosed with valvular heart disease before pregnancy develop heart failure. In our study, 5.67\% of the pregnant women with valvular heart disease developed PCHF, and had an aOR 83.79 times higher (95\% CI: 61.49-114.18) for developing PCHF than did delivering mothers without valvular heart disease.

Consistent with the findings of other studies [8], our findings showed that obstetric conditions like multifetal gestation, pre-eclampsia, and eclampsia contributed to the development of PCHF. Hypertension damages blood vessels already weakened by hemodynamic stress or altered by other factors associated with pregnancy [12].

Apart from cardiac anomalies, anemia and disorders of the pulmonary, renal, thyroidal, and autoimmune systems were also significantly associated with PCHF in this study. It is well documented that respiratory disturbances, fibrosis, and pulmonary hypertension is often comorbid with CHF secondary to right heart overload, which ultimately leads to heart failure [32]. Moreover, impaired renal function may cause fluid retention and increase cardiac workload. In addition, renal disease shares many risk factors-e.g., HTN and DM-with heart failure [33]. Thus, it is logical that renal disease increases the risk of PCHF. Similarly, in anemic patients, the heart must beat more vigorously and rapidly to meet

Table 5 Cesarean sections; length of hospital stays; and hospital cost in peripartum congestive heart failure group

\begin{tabular}{lll}
\hline Variable & $\begin{array}{l}\mathrm{PCHF} \\
n=512\end{array}$ & $\begin{array}{l}\text { No PCHF } \\
n=2,115,361\end{array}$ \\
\hline $\begin{array}{l}\text { Types of delivery } \\
\quad \text { Normal spontaneous delivery) }\end{array}$ & $172(33.59 \%)$ & $1,386,467(65.54 \%)$ \\
$\quad 340(66.41 \%)$ & $728,894(34.46 \%)$
\end{tabular}

Percentage of cesarean sections; length of hospital stays; and hospital cost in peripartum congestive heart failure group during 1997-2013 in Taiwan ${ }^{\mathrm{a}} \mathrm{PCHF}$ : peripartum congestive heart failure 
the body's demand for oxygen, which can induce cardiac de-compensation [34]. Higher levels of cytokines, in concert with higher levels of circulating autoantibodies, might attack the cardiac tissue and induce heart failure [6]. In pregnant women comorbid with thyroid problems like Graves' disease, the high-output state and additional hemodynamic burdens might further decompensate ventricular function in the peripartum period [7].

Interestingly, we also found that pregnant women comorbid with liver disease had a risk 3.61 times greater of developing PCHF than did women not comorbid with liver disease. Those with severe liver disease might experience coagulation dysfunction, anemia, and increased cardiac effort [35]. Recently, nonalcoholic fatty liver disease has been identified as an emerging risk factor for acute heart failure [36]. This might partially explain our finding about liver disease and PCHF.

We also found that delivering mothers without PCHF had greater odds of having a cesarean section than did those without. Vaginal delivery poses a potential risk of hemodynamic fluctuations related to labor pain [13]. Women at a high risk should consider an elective cesarean section. Moreover, it is reasonable that delivering mothers with PCHF tend to seek healthcare in larger and more full-service medical centers rather than in smaller local hospitals with fewer specialist physicians.

It is worth mentioning that the total peripartum hospital stay and medical costs were substantially higher for delivering mothers with than without PCHF. We think this is clinically important because it ultimately translates into a vast amount of extra medical resources. Because medical costs are increasing worldwide, the economic aspect of health care is especially important for governments that provide government subsidized national health insurance. Therefore, our results might also benefit government and health insurance policy makers.

\section{Limitations}

Our study has some limitations. First, because patient information in our database is de-identified, we could not use medical record reviews to confirm the ICD-9$\mathrm{CM}$ diagnosis and comorbidity codes. Incorrect coding and misclassification might have biased our results. However, every hospital in Taiwan has a coder team to ensure the accuracy of diagnostic and management codes. The validity of many specific diagnoses, especially those of major diseases in inpatients in Taiwan, have been verified and reported [37, 38]. Second, every patient has at most 5 ICD-9-CM diagnostic codes for each admission; therefore, minor events and comorbid disorders might not be listed at discharge. This might underestimate the association between minor diseases and PCHF. However, major diseases, such as heart failure and chronic kidney disease, would not be ignored.
Third, the medical history of patients in the NHIRD can be traced back only to January 1996 . We cannot be certain whether a pregnant woman had CHF before then, and therefore cannot exclude the possibility of mistaking a patient with prior CHF as having a new case of CHF. The prevalence of some adverse outcomes might thus be overestimated. Fourth, some important sociodemographic characteristics, such as education level, nutritional status, dietary habits, alcohol drinking and tobacco use, and BMI, are not recorded in the database. Therefore, we could not adjust for these variables as contributing factors in this study. Fifth, laboratory data and image reports, e.g., echocardiograms, and some delivery records, e.g., blood loss and hemodynamic records when giving birth, are not recorded. However, major findings are expressed as ICD-9-CM codes.

\section{Conclusion}

Maternal structural heart diseases, especially right heart and valvular heart diseases, are associated with highest risk for developing PCHF. Anemia, pulmonary, renal, and liver diseases; and preexisting and gestational HTN are also risk factors for developing PCHF. Moreover, delivering mothers with PCHF had higher ORs for adverse major maternal outcomes and cesarean sections; they also had longer hospital stays, used more medical resources, and had higher hospital costs than did delivering mothers without PCHF. Medical care providers should pay close attention to delivering mothers with high risk factors for PCHF to prevent their mortality and morbidities.

\section{Additional file}

Additional file 1: Appendix. Major Comorbidities, Obstetric Conditions, and Corresponding International Classification of Diseases, Ninth Revision, Clinical Modification Codes. Description of data: This file shows the ICD-9 CM (International Classification of Diseases, Ninth Revision, Clinical Modification) Codes used in our study. (DOC $45 \mathrm{~kb}$ )

\section{Abbreviations}

CHF: Congestive heart failure; ICD-9-CM: International Classification of Diseases, Ninth Revision, Clinical Modification; NHI: National Health Insurance; NHIRD: National Health Insurance Research Database; NT\$: New Taiwan dollars; PCHF: Peripartum congestive heart failure; PPCM: Peripartum cardiomyopathy; ROPAC: Registry of Pregnancy and Cardiac disease

\section{Acknowledgments}

This study is based on data from the Taiwan National Health Insurance Research Database provided by the Taiwan Bureau of National Health Insurance, Taiwan Department of Health, Taipei, and managed by the National Health Research Institutes (Zhunan, Miaoli County, Taiwan).

\section{Funding}

Ying-Jen Chang received a research founding (grant no. CMFHR10478) from Chi Mei Medical Center.

The remaining authors report no financial support.

Availability of data and materials

The data that support the findings of this study are available from Taiwan National Health Insurance research database. 


\section{Declarations}

This study was granted an exemption from a full ethical review by the Chi Mei Medical Center Institutional Review Board (IRB: 10,505-014).

Date of approval: 2016/06/07.

\section{Authors' contributions}

YJC reviewed the literature, designed the study, and drafted the manuscript. $\mathrm{CHH}$ helped design the study and did the statistical analyses. JYC and MPW reviewed the literature and interpreted the results. CHY helped do the statistical analyses. JJW revised the manuscript and provided administrative and technical support. CCC and CMC conceived and helped design the study, coordinated and interpreted the results, and revised the manuscript. CCC and CMC contributed equally to this study. All authors have read and approved the final version of this manuscript.

\section{Authors' information}

Both Chia-Ming Chen (M.D.) and Chin-Chen Chu (M.D., Ph.D.) are senior anesthesiologists at Chi Mei Medical Center. Chu is a professor and works as the director of the division of women's and children's anesthesia.

\section{Consent for publication}

Not applicable. This is an observational nationwide population database study.

\section{Competing interests}

The authors declare that they have no competing interests.

\section{Publisher's Note}

Springer Nature remains neutral with regard to jurisdictional claims in published maps and institutional affiliations.

\section{Author details}

'Department of Anesthesiology, Chi Mei Medical Center, 901 Zhonghua Road, Yongkang District, Tainan City 710, Taiwan. ${ }^{2}$ Department of Medical Research, Chi Mei Medical Center, 901 Zhonghua Road, Yongkang District, Tainan City 710, Taiwan. ${ }^{3}$ Department of Pharmacy, Chia Nan University of Pharmacy and Science, Tainan, Taiwan. ${ }^{4}$ Department of Senior Citizen Service Management, Chia Nan University of Pharmacy and Science, Tainan, Taiwan. ${ }^{5}$ Division of Urogynecology and Pelvic Floor Reconstruction, Department of Obstetrics and Gynecology, Chi Mei Medical Center, 901 Zhonghua Road, Yongkang District, Tainan City 710, Taiwan. ${ }^{6}$ Center of General Education, Chia Nan University of Pharmacy and Science, Tainan, Taiwan. ${ }^{7}$ Department of Recreation and Health-Care Management, Chia Nan University of Pharmacy and Science, Tainan, Taiwan. ${ }^{8}$ Division of Women's and Children's Anesthesia, Department of Anesthesiology, Chi Mei Medical Center, 901 Zhonghua Road, Yongkang District, Tainan City 710, Taiwan.

\section{Received: 7 December 2016 Accepted: 4 September 2017}

\section{Published online: 12 September 2017}

\section{References}

1. Francis GS, Tang WH. Pathophysiology of congestive heart failure. Rev Cardiovasc Med. 2003;4(Suppl 2):S14-20

2. Koutrolou-Sotiropoulou P, Parikh PB, Miller C, Lima FV, Butler J, Stergiopoulos K. Impact of heart disease on maternal and fetal outcomes in pregnant women. Am J Cardiol. 2015;116(3):474-80.

3. Mhyre JM, Tsen LC, Einav S, Kuklina EV, Leffert LR, Bateman BT. Cardiac arrest during hospitalization for delivery in the United States, 1998-2011. Anesthesiology. 2014;120(4):810-8.

4. Ruys TP, Roos-Hesselink JW, Hall R, Subirana-Domenech MT, Grando-Ting J, Estensen $\mathrm{M}$, et al. Heart failure in pregnant women with cardiac disease: data from the ROPAC. Heart. 2014;100(3):231-8.

5. Wu LS, Liu PH, Lin YS, Lin CP, Chang CJ, Chu PH. Lupus women with delivery with higher risk of heart failure compared with those without pregnancy but neutral in major adverse cardiovascular events. A population-based matched cohort study. Clin Exp Rheumatol. 2014:32(1):108-12.

6. Ansari AA, Fett JD, Carraway RE, Mayne AE, Onlamoon N, Sundstrom JB. Autoimmune mechanisms as the basis for human peripartum cardiomyopathy. Clin Rev Allergy Immunol. 2002;23(3):301-24

7. Sheffield JS, Cunningham FG. Thyrotoxicosis and heart failure that complicate pregnancy. Am J Obstet Gynecol. 2004;190(1):211-7.
8. Ray JG, Vermeulen MJ, Schull MJ, Redelmeier DA. Cardiovascular health after maternal placental syndromes (CHAMPS): population-based retrospective cohort study. Lancet. 2005;366(9499):1797-803.

9. Hilfiker-Kleiner D, Sliwa K. Pathophysiology and epidemiology of peripartum cardiomyopathy. Nat Rev Cardiol. 2014;11(6):364-70.

10. Givertz MM. Cardiology patient page: peripartum cardiomyopathy. Circulation. 2013;127(20):e622-6.

11. McNamara DM, Elkayam U, Alharethi R, Damp J, Hsich E, Ewald G, et al. Clinical outcomes for peripartum cardiomyopathy in North America: results of the IPAC study (Investigations of Pregnancy-Associated Cardiomyopathy). J Am Coll Cardiol. 2015;66(8):905-14.

12. James AH, Jamison MG, Biswas MS, Brancazio LR, Swamy GK, Myers ER. Acute myocardial infarction in pregnancy: a United States population-based study. Circulation. 2006;113(12):1564-71.

13. Goland S, Bitar F, Modi K, Safirstein J, Ro A, Mirocha J, et al. Evaluation of the clinical relevance of baseline left ventricular ejection fraction as a predictor of recovery or persistence of severe dysfunction in women in the United States with peripartum cardiomyopathy. J Card Fail. 2011;17(5):426-30.

14. Rutherford JD. Heart failure in pregnancy. Curr Heart Fail Rep. 2012;9(4):277-81.

15. Hsich EM, Pina IL. Heart failure in women: a need for prospective data. J Am Coll Cardiol. 2009;54(6):491-8.

16. Ntusi NB, Badri M, Gumedze F, Sliwa K, Mayosi BM. Pregnancy-associated heart failure: a comparison of clinical presentation and outcome between hypertensive heart failure of pregnancy and idiopathic peripartum cardiomyopathy. PLoS One. 2015;10(8):e0133466.

17. Chen L, Yip W, Chang MC, Lin HS, Lee SD, Chiu YL, et al. The effects of Taiwan's National Health Insurance on access and health status of the elderly. Health Econ. 2007;16(3):223-42.

18. Universal Health Coverage in Taiwan. National Health Insurance Administration, Ministry of Health and Welfare, Taiwan. 2016. http://www. nhi.gov.tw/Resource/webdata/21717_1_UnversalHealthCoverage-2.pdf.

19. Demakis JG, Rahimtoola SH. Peripartum cardiomyopathy. Circulation. 1971; 44(5):964-8

20. Sliwa K, Bohm M. Incidence and prevalence of pregnancy-related heart disease. Cardiovasc Res. 2014;101(4):554-60.

21. Bhattacharyya A, Basra SS, Sen P, Kar B. Peripartum cardiomyopathy: a review. Tex Heart Inst J. 2012;39(1):8-16.

22. Capriola M. Peripartum cardiomyopathy: a review. Int J Womens Health. 2013;5:1-8.

23. Kamiya CA, Kitakaze M, Ishibashi-Ueda H, Nakatani S, Murohara T, Tomoike $\mathrm{H}$, et al. Different characteristics of peripartum cardiomyopathy between patients complicated with and without hypertensive disorders. -Results from the Japanese Nationwide survey of peripartum cardiomyopathy. Circ J. 2011;75(8):1975-81.

24. Dickinson JE. Increasing maternal age and obstetric outcomes. Aust N Z J Obstet Gynaecol. 2012;52(3):217-9.

25. Carolan M. Maternal age $>/=45$ years and maternal and perinatal outcomes: a review of the evidence. Midwifery. 2013;29(5):479-89.

26. Roos-Hesselink JW, Ruys TP, Stein J, Thilen U, Webb GD, Niwa K, et al. Outcome of pregnancy in patients with structural or ischaemic heart disease: results of a registry of the European Society of Cardiology. Eur Heart J. 2013;34(9):657-65.

27. Drenthen W, Pieper PG, Roos-Hesselink JW, van Lottum WA, Voors AA, Mulder BJ, et al. Outcome of pregnancy in women with congenital heart disease: a literature review. J Am Coll Cardiol. 2007:49(24): 2303-11.

28. Hung $\mathrm{TH}$, Hsieh $\Pi$. Pregestational body mass index, gestational weight gain, and risks for adverse pregnancy outcomes among Taiwanese women: a retrospective cohort study. Taiwan J Obstet Gynecol. 2016; 55(4):575-81.

29. Allen AM, Kim WR, Larson JJ, Rosedahl JK, Yawn BP, McKeon K, et al. The epidemiology of liver diseases unique to pregnancy in a US community: a population-based study. Clin Gastroenterol Hepatol. 2016;14(2):287-94 e281-2.

30. Siu SC, Sermer M, Colman JM, Alvarez AN, Mercier LA, Morton BC, et al. Prospective multicenter study of pregnancy outcomes in women with heart disease. Circulation. 2001;104(5):515-21.

31. Fu Q, Lin J. Risk factors for heart failure during pregnancy among Chinese women with cardiac disease. Int J Gynaecol Obstet. 2015;130(3):266-9.

32. Dayeh NR, Ledoux J, Dupuis J. Lung capillary stress failure and arteriolar Remodelling in pulmonary hypertension associated with left heart disease (Group 2 PH). Prog Cardiovasc Dis. 2016;59(1):11-21. 
33. Ahmed A, Campbell RC. Epidemiology of chronic kidney disease in heart failure. Heart Fail Clin. 2008;4(4):387-99.

34. Coats AJ. Anaemia and heart failure. Heart. 2004;90(9):977-9.

35. Castro MA, Fassett MJ, Reynolds TB, Shaw KJ, Goodwin TM. Reversible peripartum liver failure: a new perspective on the diagnosis, treatment, and cause of acute fatty liver of pregnancy, based on 28 consecutive cases. Am J Obstet Gynecol. 1999;181(2):389-95.

36. Valbusa F, Bonapace S, Agnoletti D, Scala L, Grillo C, Arduini P, et al. Nonalcoholic fatty liver disease and increased risk of 1-year all-cause and cardiac hospital readmissions in elderly patients admitted for acute heart failure. PLoS One. 2017;12(3):e0173398.

37. Cheng CL, Kao YH, Lin SJ, Lee CH, Lai ML. Validation of the National Health Insurance Research Database with ischemic stroke cases in Taiwan. Pharmacoepidemiol Drug Saf. 2011;20(3):236-42.

38. Cheng CL, Lee CH, Chen PS, Li YH, Lin SJ, Yang YH. Validation of acute myocardial infarction cases in the national health insurance research database in taiwan. J Epidemiol. 2014;24(6):500-7.

Submit your next manuscript to BioMed Central and we will help you at every step:

- We accept pre-submission inquiries

- Our selector tool helps you to find the most relevant journal

- We provide round the clock customer support

- Convenient online submission

- Thorough peer review

- Inclusion in PubMed and all major indexing services

- Maximum visibility for your research

Submit your manuscript at www.biomedcentral.com/submit
Biomed Central 